\title{
Patients' pain experience following oral mucosal biopsy under local anaesthesia
}

\author{
H. P. O. Kearns, ' B. E. McCartan, ${ }^{2}$ and P-J. Lamey, ${ }^{3}$
}

\begin{abstract}
Objective To examine the incidence and severity of postoperative pain and use of analgesics following biopsy of oral mucosal lesions.

Design A patient survey using a self-completed pain diary. Subjects and Methods Seventy-six patients attending an oral medicine clinic for investigation of oral mucosal disease recorded overall and worst pain experiences and analgesic usage over 7 post-operative days following biopsy using visual analogue scales in patient completed diaries.

Results One third of patients reported no post-operative pain on any day. Most patients recorded no pain or mild pain. The percentage experiencing pain dropped from the first day $(61 \%)$ to the seventh day $(21 \%)$. Most patients did not use any analgesics. Analgesic use was not related to the presence of pain. Only a small minority of patients recorded severe pain.

Conclusion Biopsy of oral mucosal lesions does not cause undue pain in most patients.
\end{abstract}

$\mathbf{B}$ iopsy of lesions of the oral mucous membranes is a common Bminor surgical procedure, usually performed under local anaesthesia. Although previous workers have investigated pain, swelling and trismus following surgical removal of teeth ${ }^{1,2}$ there do not appear to be any reports on pain experience in patients with healing wounds following oral mucosal biopsy. Similarly, there appear to be no reports in the literature of pain following dermatological and gynaecological soft tissue biopsies. This may be, in part, caused by the subjective nature of pain and the inherent problems of measuring a continuous subjective response over a period of time.

Previous studies of post-operative pain following surgical removal of third molars have shown that there is no relationship between the operative trauma and the magnitude of the post-operative pain. 1,3,4 The pain experienced following third molar surgery under local anaesthesia has been shown to be of short duration and reaches its maximum intensity in the early post-operative period. ${ }^{1}$ The same workers found that the magnitude of post-operative pain does not appear to be related to the operator who carried out the surgery. There are conflicting reports on whether a relationship exists between sex and pain experience. . $^{1,3,4}$

Soft tissue biopsy of oral lesions is often necessary to obtain a

${ }^{1}$ Research Fellow in Oral Medicine, Department of Oral Surgery, Oral Medicine and Oral Pathology, School of Dental Science, Trinity College Dublin, Dublin 2, Ireland; ${ }^{*}$ Senior Lecturer/Consultant in Oral Medicine, Department of Oral Surgery, Oral Medicine and Oral Pathology, School of Dental Science, Trinity College Dublin, Dublin 2, Ireland; ${ }^{3}$ Professor of Oral Medicine, School of Clinical Dentistry, The Queen's University of Belfast, Grosvenor Road, Belfast BT12 6BP, United Kingdom

${ }^{\star}$ Correspondence to: B. E. McCartan, Department of Oral Surgery, Oral Medicine and Oral Pathology, School of Dental Science, Trinity College, Dublin 2, Ireland email:bmccrtan@dental.tcd.ie

REFEREED PAPER

Received 21.01.00; accepted 26.06.00

(C) British Dental Journal 2000; 190: 33-35 definitive diagnosis. A clear understanding of the pain experience associated with soft tissue biopsies would aid clinicians in preparing patients for these procedures. The purpose of this study was to examine the incidence and severity of post-operative pain and the use of analgesics following incisional and excisional biopsy of lesions of the oral mucous membranes.

\section{Subjects and methods}

Eighty-five consecutive patients, aged 15 years or over, undergoing biopsy of lesions of the oral mucous membranes under local anaesthesia in an oral medicine clinic were recruited into the study. Informed consent was obtained for each biopsy. Each patient was given a pain diary to complete for each of the 7 post-operative days. Patients were instructed to note 3 items for each day: the overall level of pain, the worst pain experienced and whether analgesics had been taken. Overall and worst pain levels were recorded using visual analogue scales (VAS), each consisting of a $100 \mathrm{~mm}$ calibrated line on white paper, marked 0 to 10 at $10 \mathrm{~mm}$ intervals. Patients were informed that a score of 0 indicated 'no pain at all' while a score of 10 indicated 'the pain could not be worse'. Patients were instructed to record in the diary each morning the pain experience of the previous day, by marking an appropriate point on the VAS for worst pain and an appropriate point on the VAS for overall pain and by answering 'yes' or 'no' to the question on analgesic use. Records were also kept of age, sex and biopsy site. Pain scores were further categorised as 'none', 'mild', 'moderate', or 'severe' using previously established criteria, ${ }^{5}$ zero scores on the VAS being regarded as 'no pain', 1-3 as 'mild' pain, 4-6 as 'moderate' and 7-10 as 'severe'. Patients were reviewed on completion of the 7-day diary to ensure normal healing and absence of infection.

\section{Results}

Nine returned diaries out of the 85 were incorrectly completed and were discarded from the study. Most failures resulted from incorrect use of the VAS by the patient. Thus, 76 diaries were available for analysis. Clinically no biopsied area had become infected and all biopsy sites were seen to be healing normally.

Twenty-eight of the patients (37\%) were men and 48 (63\%) were women. The median age was 51 years (IQR 33 to 65 ), range 15 to 85 years. The sites of biopsy are shown in Table 1 . Twentyfive patients $(33 \%)$ recorded no pain at all on any day. On the first day 30 patients (39\%) recorded no pain; this rose to 60 patients $(79 \%)$ by the seventh day (Table 2$)$. On day one 59 patients $(78 \%)$ recorded overall pain levels corresponding to none or mild and $55(72 \%)$ recorded worst pain levels corresponding to none or mild. The corresponding figures for the seventh day were $72(95 \%)$ and $72(95 \%)$ (see Table 3). Out of a total of 1064 VAS scores, only $110(10 \%)$ corresponded to moderate or severe pain and only $38(4 \%)$ corresponded to severe pain; pain scores corresponding to severe pain were recorded by only 11 patients (14\%). The experiences recorded for overall and worst pain were similar; while there were some differences 
Table I Site distribution of biopsies with analgesic use and pain experience for each site

Site No Per cent $\begin{gathered}\begin{array}{c}\text { Per cent taking } \\ \text { analgesics } \\ \text { anytime }\end{array} \\ \text { nntime }\end{gathered}$

$\begin{array}{lrrr}\text { Buccal mucous membranes } & 38 & 50 & 34 \\ \text { Lip } & 12 & 16 & 17 \\ \text { Palate } & 10 & 13 & 30 \\ \text { Tongue } & 7 & 9 & 29 \\ \text { Gingiva/alveolus } & 6 & 8 & 50 \\ \text { Floor of mouth } & 3 & 4 & 0\end{array}$

Table 2 Numbers of patients pain free on each postoperative day on both overall and worst VAS

\begin{tabular}{llllllll}
\hline & & & & Day & & & \\
& 1 & 2 & 3 & 4 & 5 & 6 & 7 \\
\hline Number & 30 & 40 & 42 & 51 & 52 & 58 & 60 \\
Per cent & 39 & 53 & 55 & 67 & 68 & 76 & 79 \\
\hline
\end{tabular}

between the two VAS scores, in only $5 \%$ of cases did this correspond to a different pain category.

Fifty-three patients (70\%) did not take any analgesics on any day; a further 11 patients (15\%) used analgesics on the first day only. Twenty patients (26\%) took analgesics on the first day compared with only $2(3 \%)$ on the seventh day (Table 4$)$. A substantial number of patients with mild pain or no pain took analgesics while a small number of patients with severe pain did not. When the 25 patients who did not experience pain on any day were excluded, analgesic use mirrored both overall and worst pain in women while men with low pain scores were likely not to use analgesics.

\section{Discussion}

A subjective stimulus such as pain is difficult to measure accurately as it is often accompanied by other sensations and altered by peripherally and centrally acting analgesics, and also because of variations in patient preparedness to report the level of pain.

There are a number of ways of measuring pain experience. One simple but imprecise method is to measure the dose of analgesics required to obtain a subjective reduction of 'more than half' of pain in individual subjects. ${ }^{6}$ Another simple method of low sensitivity is to ask patients whether they are having, or have had, pain. ${ }^{7}$ More sensitive methods include the McGill Pain Questionnaire, ${ }^{8}$ which has been shown to be a sensitive method of recording both type and intensity of pain. However, the use of the McGill Pain Questionnaire is dependent on patient understanding of the many verbal descriptions of pain and we that felt its repeated use on each of 7 consecutive days would be too complex for many of our patients.

The visual analogue scale (VAS) is a simple method of selfrating of subjective sensations including pain. ${ }^{9,10}$ The analogue scale acts as a pain thermometer, the patient being offered a continuous scale for evaluation of pain and the VAS has been shown to be a suitable method for recording post-operative dental pain ${ }^{11}$ and is reliable in use. ${ }^{12-14}$ Even so we had a failure rate of $11 \%$ of incorrectly completed diaries despite careful patient instruction. We have recently shown that there is an equivalence in dental pain between recordings made with VAS and with verbal descriptors ${ }^{5}$ and we were able to apply those equivalent values to the findings, allowing us to categorise the pain experiences. Pain measurements should, ideally, be contemporary. Measuring pain after the event is not accurate, the problem of faulty recall being added to the subjective nature of the measurement. However, there is some evidence that recall after the event is accurate. ${ }^{12}$ As the aim of this study was to record pain experience over each of the 7 post-operative days rather than at specific times during each day, it was felt that the problems inherent in reliance on patient recall would have to be accepted; the results must be interpreted in that light. To minimise errors which might arise, each patient was carefully instructed in the format of the diary and its correct use.

The findings suggest that many patients are either completely pain free or suffer only mild pain following biopsy under local anaesthesia of oral mucous membrane lesions. In most patients who experienced more severe pain, the severity of pain reduced considerably after the first 3 post-operative days. Three patients continued to experience severe pain after day three. The study design does not permit us to comment fully on these cases but we presume that they were either patients whose oral condition was painful in itself or that they were patients with low pain thresholds.

The reduction in pain scores over the 7 days was matched by a reduction in the number of patients taking analgesics. The consumption of analgesics will alter the nature and duration of the pain experience reported. While a definitive view of post-operative pain could only be obtained by instructing patients to abstain from taking analgesics, this would be unethical, and to do so would not demonstrate the true clinical picture. Compliance with

Table 4 Numbers of patients taking analgesics on each postoperative day

\begin{tabular}{lccccccc}
\hline & 1 & 2 & 3 & 4 & 5 & 6 & 7 \\
\hline Number & 20 & 10 & 9 & 5 & 3 & 4 & 2 \\
Per cent & 26 & 13 & 12 & 7 & 4 & 5 & 3 \\
\hline
\end{tabular}

Table 3 Categorisation of pain experience on each postoperative day.

\begin{tabular}{|c|c|c|c|c|c|c|c|c|c|}
\hline & & & & & & & & & \\
\hline & & I & 2 & 3 & 4 & 5 & 6 & 7 & I-7 \\
\hline Category & & & & & & & & & \\
\hline None & Overall & 31 & 40 & 43 & 52 & 52 & 59 & 62 & 339 \\
\hline & Worst & 32 & 41 & 42 & 51 & 52 & 58 & 60 & 336 \\
\hline Mild & Overall & 28 & 27 & 26 & 21 & 20 & 12 & 10 & 144 \\
\hline & Worst & 23 & 25 & 25 & 20 & 18 & 12 & 12 & 135 \\
\hline Moderate & Overall & 14 & 8 & 5 & 2 & 3 & 3 & 2 & 37 \\
\hline & Worst & 12 & 6 & 6 & 4 & 3 & 3 & I & 35 \\
\hline Severe & Overall & 3 & I & 2 & I & I & 2 & 2 & 12 \\
\hline & Worst & 9 & 4 & 3 & I & 3 & 3 & 3 & 26 \\
\hline
\end{tabular}


a regime of non-use of analgesics would probably be poor, further compromising the results. A small number of patients continued to take analgesics throughout the 7 days. These patients' pain experience on these days varied from none to severe. It seems that a small number of patients continued to take prophylactic analgesics although experiencing no pain.

\section{Conclusion}

The study has shown that pain after oral mucous membrane biopsy is absent or mild in most patients. This information should be of use to those who have to advise patients of the necessity of such biopsies in the investigation of oral disease.

1 Seymour R A, Meechan J G, Blair G S. An investigation into post-operative pain after third molar surgery under local anaesthesia. Br J Oral Maxillofac Surg 1985; 23: 410-8.

2 Holland C S. The development of a method of assessing swelling following third molar surgery. Br J Oral Surg 1979; 17: 104-114.

3 van Buren J, Kleinknecht R A. An evaluation of the McGill Pain Questionnaire for use in dental pain assessment. Pain 1979; 6: 23-33.

4 Seymour R A, Blair G S, Wyatt F A R. Post-operative dental pain and analgesic efficacy. Part 1. Br J Oral Surg 1983; 21: 290-297.

5 McCartan B E, Kearns H P O, Lamey P-J. Verbal descriptors in the measurement of dental pain. Pain Clinic 1997; 10: 35-37.
6 Beecher H K. Pain and some factors that modify it. Anesthesiol 1951; 12: 633-641.

7 MacGregor A J, Hart P. Effect of bacteria and other factors on pain and swelling after removal of ectopic mandibular third molars. J Oral Surg 1969; 27: 174-179.

8 Melzack R. The McGill Pain Questionnaire: major properties and scoring methods. Pain 1975; 1: 277-99.

9 Clarke P R F, Spear F Q. Reliability and sensitivity in the self assessment of well being. Bull Br Psychol Soc 1964; 17: 18A.

10 Bond M R, Pilowski I. The subjective assessment of pain and its relationship to the administration of analgesics in patients with advanced cancer. J Psychosom Res 1966; 10: 203-208.

11 Seymour R A. Dental pain. 3. The measurement of pain. Dent Update 1983; 10: 446-454.

12 Revill S I, Robinson J O, Rosen M, Hogg M I. The reliability of a linear analogue for evaluating pain. Anaesthesia 1976; 31: 1191-1198.

13 Ekowski C, Hrubes V, Joyce C R B, Zutshi D W, Flood M K, Mason R M. An experimental study of two methodological problems in clinical evaluation: different types of scale and the availability of patients' previous judgements. Psychopharmacol (Suppl) 1972; 26: 70.

14 Ohnhaus E E, Adler R. Methodological problems in the measurement of pain: a comparison between the verbal rating scale and the visual analogue scale. Pain 1975; 1: 379-384.

15 Joyce C R B, Zutshi D W, Hrubes V, Mason R M. Comparison of fixed interval and visual analogue scales for rating chronic pain. Eur J Clin Pharmacol 1975; 8: 415-420. 